\title{
Smart Monitoring for Soldier Health and Location
}

\author{
Eman Serag, Mahmoud Mohamed Ahmed Sayed, Mahmoud Hanafy
}

Engineering Department, Canadian Internal College, Cairo, Egypt

\section{Email address:}

eman_serag@cic-cairo.com (E. Serag),m_m_sayed@cic-cairo.com (M. M. A. Sayed), mahmoud_hanafy@cic-cairo.com (M. Hanafy)

\section{To cite this article:}

Eman Serag, Mahmoud Mohamed Ahmed Sayed, Mahmoud Hanafy. Smart Monitoring for Soldier Health and Location. American Journal of Embedded Systems and Applications. Vol. 5, No. 6, 2017, pp. 48-53. doi: 10.11648/j.ajesa.20170506.12

Received: November 27, 2017; Accepted: December 12, 2017; Published: January 11, 2018

\begin{abstract}
The people around the world live a safe life due to the sacrifices of the brave soldiers, in order repay the favor, so we must shield them from risks due to they face in the warfare by equipping them with advanced technology. Equipping people especially soldiers to guarantee the security of the state and its stability. Terrorism in a lot area around the world (Sinai Peninsula that is considered one of the dangerous area) is represented threat on people life. This work designed smart monitoring system for soldiers who protecting the homeland so, it is necessary to help them by using smart monitoring system to avoid any terrorist attack or know their places when the abduction of any one of these soldiers. Supply soldier with modern technological devices makes it easy for us to know the health status and their location and this makes the control rooms in the military full-time to monitor the enemy rather than preoccupation with monitoring soldiers. Wireless communications devices play an important role in monitoring the soldiers through the use of the devices Global Positioning System (GPS) system, and also SOS messages that help the soldier to adapt with different situation. All the data collected from the sensors and send to the web server to make analysis also statistics depending on these information Base Stations can make the right decision and send it to the soldier to follow thanks to this smart control system.
\end{abstract}

Keywords: GPS, GSM, Heartrate Sensor, Temperature Sensor, Oxygen Sensor, Vibration, Microcontrolle

\section{Introduction}

The role of some people critical when they preform is considered one of important factors in any nation's security, which is represented as protection of the state in both internally and externally. As a result the proposed system will protect them from risk that they face in the warfare by equipping the soldier with smart technology to monitor their health and position.

By modern technology to identify and enable the base station to make statistics to give depending on these analyses to monitor its health status and allocation the proper order which suit the situation make the right decision and send it to the soldier to follow [1].

In this work, the system is offering a technology which provide more safety by monitoring the soldier health status, light to carry and system made especially for soldier who involve in special operation or mission. Thanks to wireless communication systems, Base Station can track the soldier position in case that need help thanks to the GPS modem also the health status to know soldier case, also provide SOS messages that soldier can inform its condition. Depending on this smart monitoring system using web server with friendly user interface based on UI and UX theories not that but also secure which enables the Base Station to give orders that how the operation will going on these orders displayed on LCD with vibration alert soldier will be able to notification the pay attention to the order [2].

Provide an additional layer of safety, RFID tag as necklace to verify soldier identity, light weight, easy to carry, temperature sensor to senesce the soldier temperature, heart beat sensor to get heart rate, GSM module send data also send SMS if the SOS button pressed and, GPS module to locate the soldier, Base Station based on Linux webserver to collect data also analysis it to get statistic, easy to use and enhance security features, also can send orders to soldier, displays on the soldier LCD with vibration actuator to make the soldier pay attention to the new orders to keep the soldier with. Also at any case soldier have SOS messages that help the soldier to adapt with different situation. All these features to help soldier to get over limitation and give soldier more abilities, safety.

Smart systems can track the soldiers at any moment to support the decision makers for evaluating any situation [7-10]. 
This paper is organized as follows: the Base station part in Section 2. The soldier unit part in Section 3. Implementing the information security triad to enhance the proposed system security features in section 4 , the simulation results are shown in the form of the GUI website based. Section 5 is devoted to conclusion. Reference will be represented in Section 6.

\section{Base Station}

With an interface a webpage design coded by (HTML5, CSS3, JQuery, Bootstrap) easy to Base Station to manage with friendly graphical user interface, with abilities that can be open from any device from computer till smartphone and tablets, also provide good experience UX to provide best way to inter act with it in an easy way. as a military project security is a vital part of the system, based on well-known open source OS Linux also secure with PHP encryption method that required username and password that are hashed in MD5 hash. [3], in order to enter the webpage. With MySQL data base in table forms that arrange every soldier and give the Base Station many features such as make full report and statistics from collected information as a proper or full monitoring system. Which enable the Base Station to take the statistics to put the right plan for the mission. Data to connect it to webserver (Apache) and data base (MySQL) to immediately rearrange it and put it in the database tables. using the organization that responsible for web W3. [4] as shown in figure 1 .

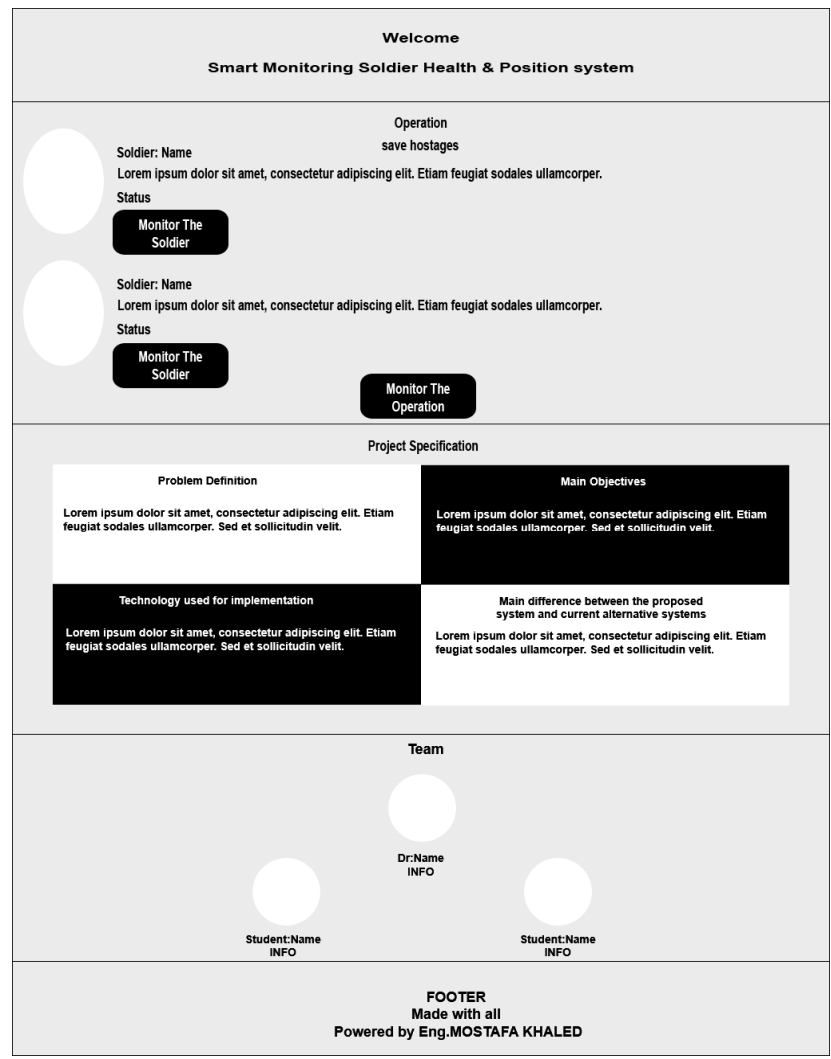

Figure 1. Base Station website Wireframe. implement. Wireframe consists of five parts that describes the Base Station website, first part is the header that includes the welcoming heading and the project title, second part is the most important part consists of the operation and the smart solider monitoring system the core of this project is in this part, also you can monitoring soldier by soldier (individual) select to monitoring or select the whole operation to monitoring in this part you can give orders to one or more soldier, also you have the ability to send orders to the whole operation (all the soldiers that participated in the operation) and you can monitor the heath and position of the selected option not that but also monitoring if they in dangerous (emergency) situation or need supplies by the bush buttons with the soldiers, third part is the theory about the project includes problem definition, main objectives, technology used for implementation, main difference between the proposed system and current alternative systems, briefly, fourth part includes the team work that cooperate to achieve this proposed system together, last part is the footer here where the copyright of this project appears.

After preparing the wireframe and learning the needed languages

(HTML5-CSS3-JQUERY-BOOTSTRAP-PHP-SQL) to make this wireframe real and make sure this website tested, provide high performance, stability, secure, validate form the W3, responsive, and provide the required task in proper way. The Base Station website shown in figure 2.

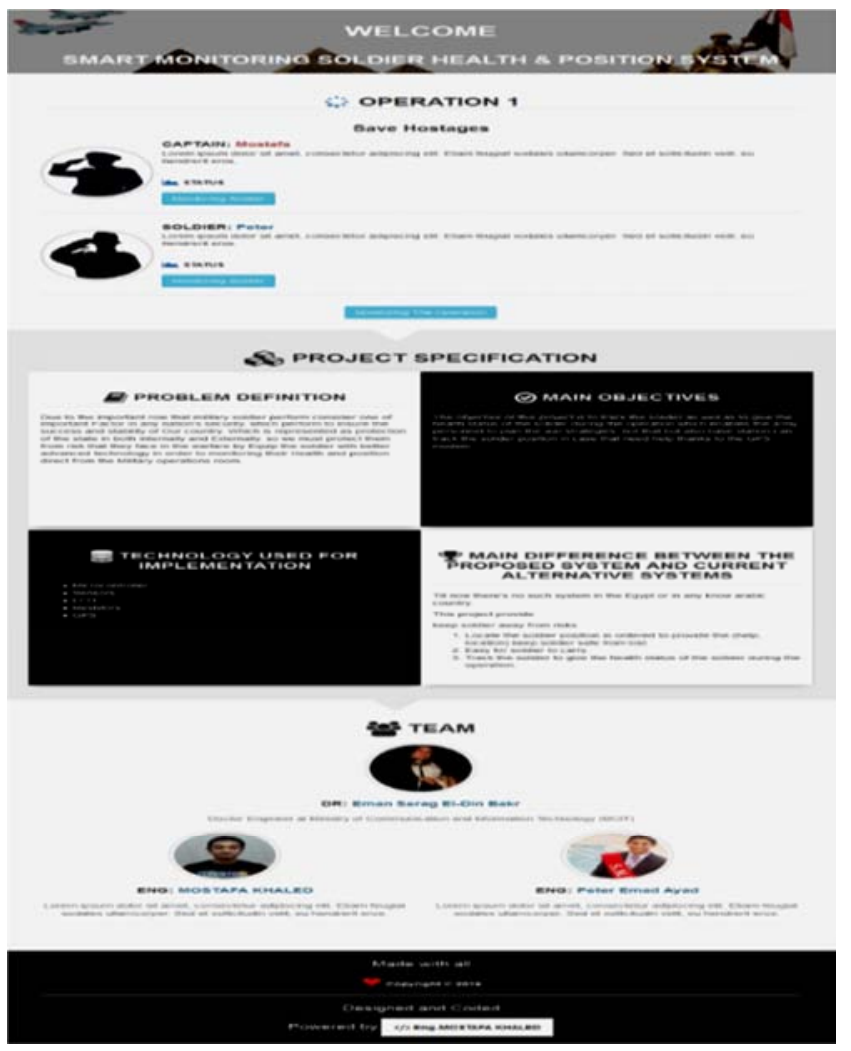

Figure 2. Base Station website.

Wireframe illustrates Base Station shape that is going to 


\section{Soldier Unit}

Soldier unit can monitor the heart beat count and temperature of soldiers by using wireless body area sensor networks [7].

The soldier unit part consists of microcontroller unit, sensors, vibration, RFID, LCD, SOS, and GPS, GSM module which is the part the soldier is going to carry to monitor health status and locate the position of the soldier. This helps the soldier to get beyond the limitation, providing features such as light weight, small size, easy to carry, waterproof. Solider unit block diagram shown in figure 3 .

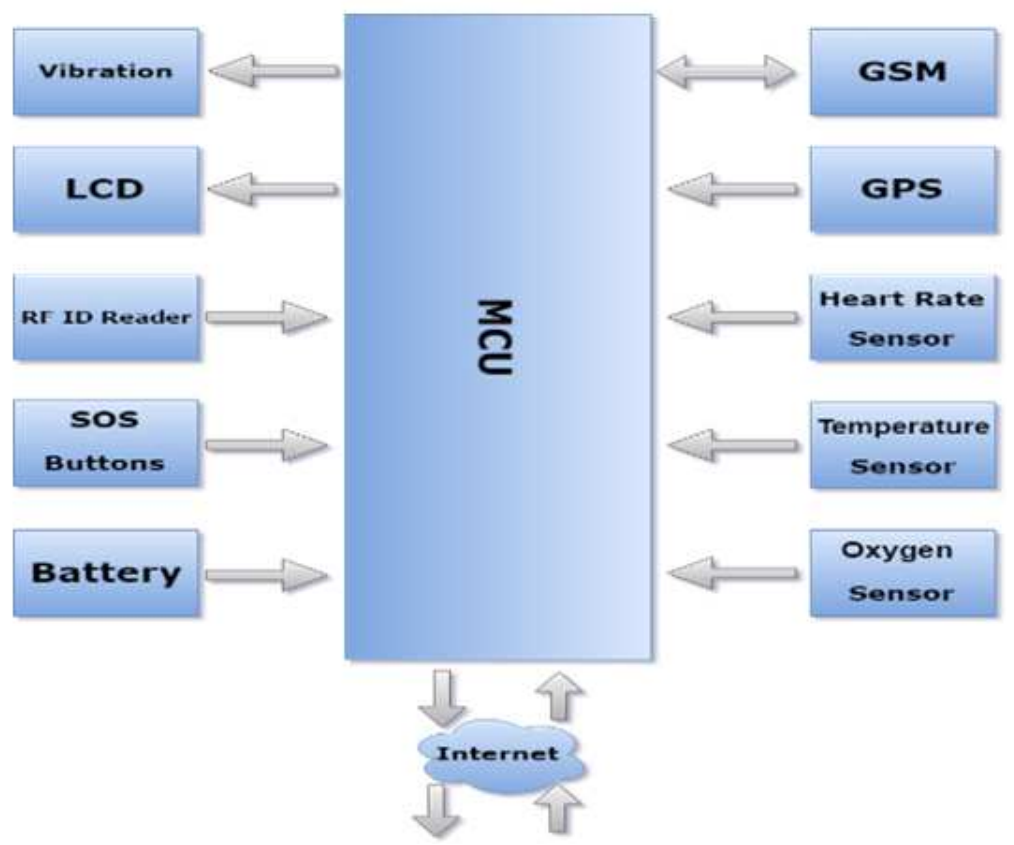

Figure 3. Soldier Unit Block diagram.

System design shades the light on designing of the system Circuit diagram which it contains detailed circuit diagram of each individual module such as the microcontroller, GSM,
GPS, Sensors, LCD another components such as RFID, resistances, regulators, and power management system. Can be shown in figure 4 .

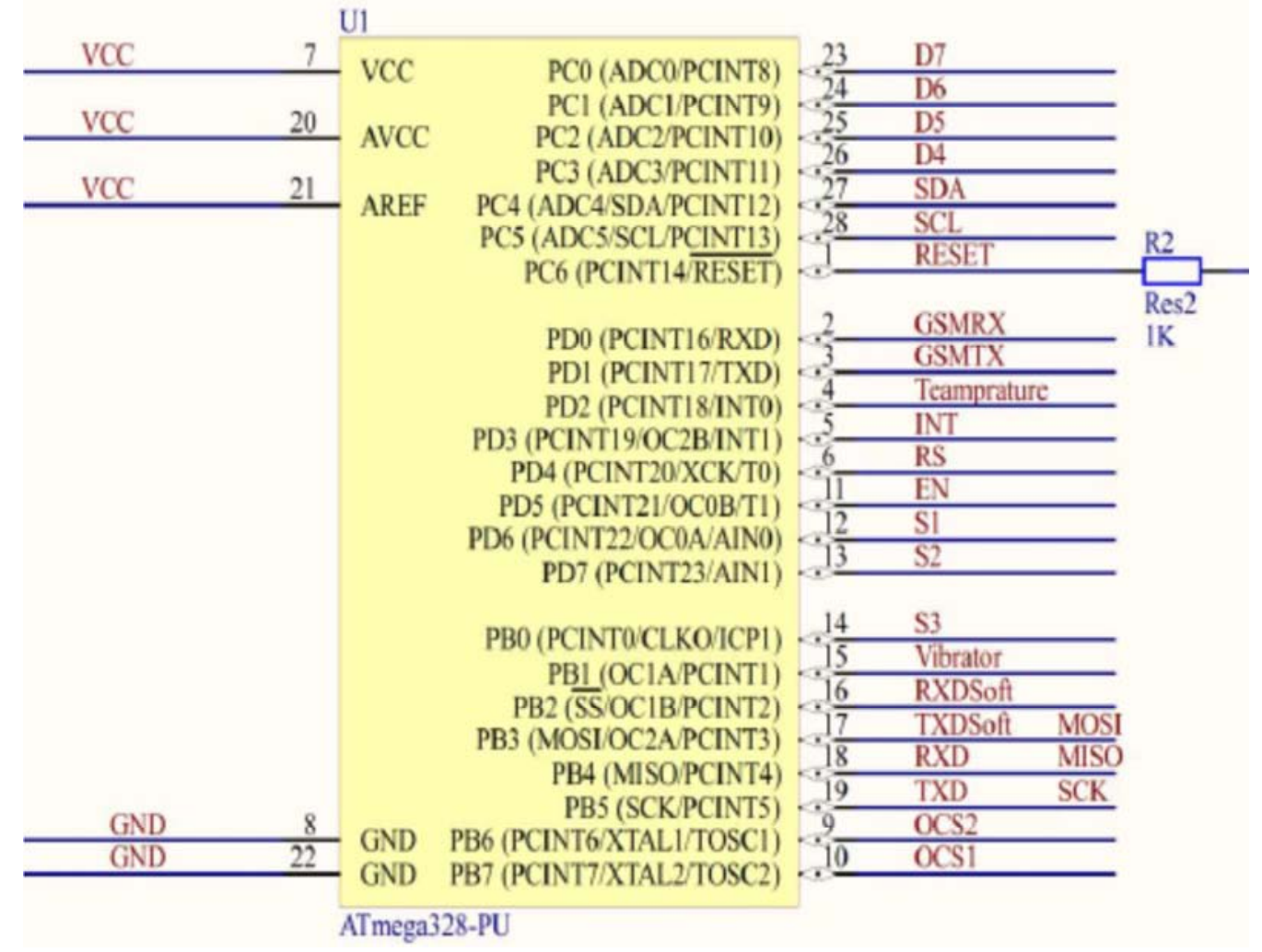



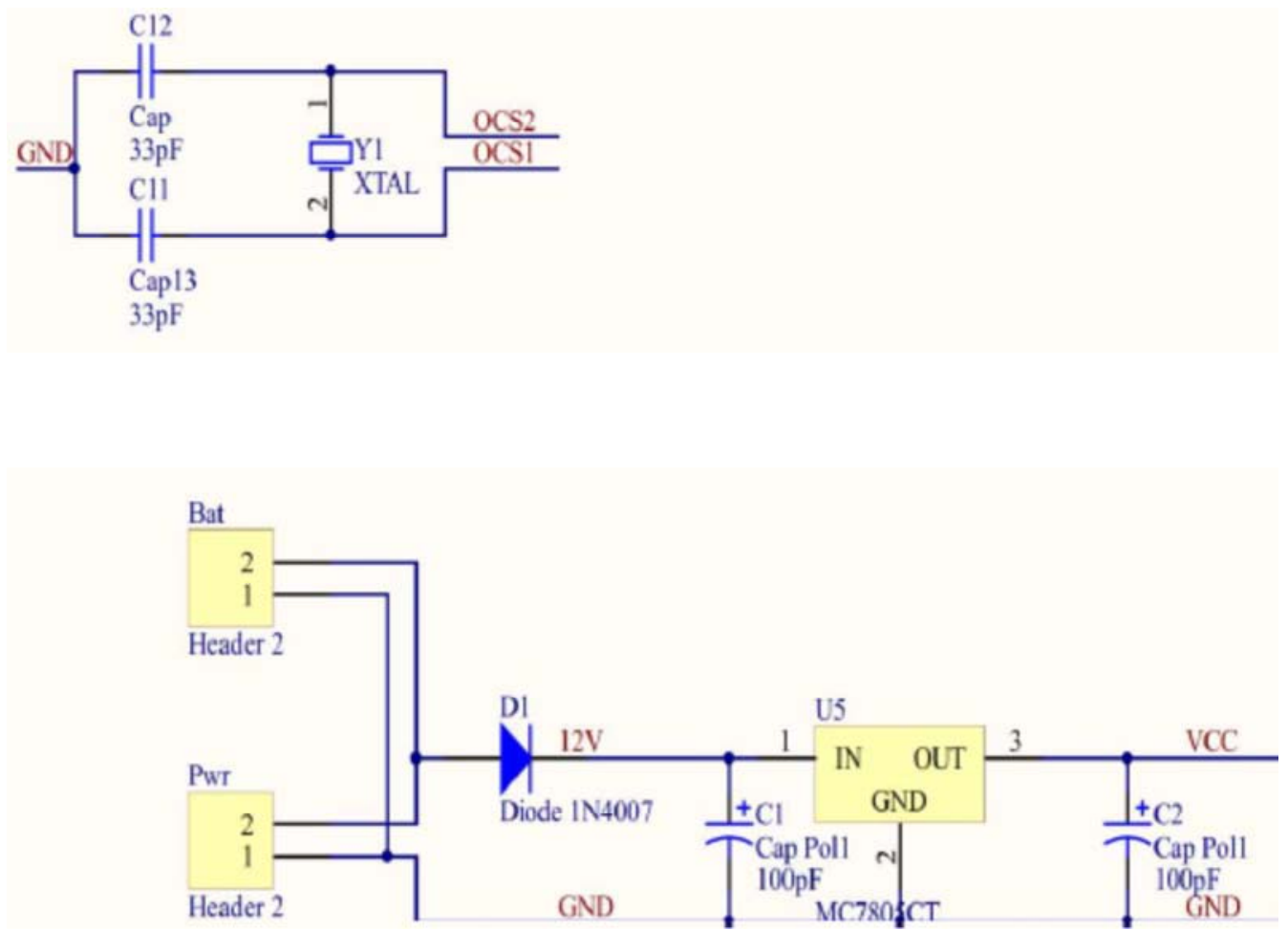

Figure 4. Circuit diagram.

\section{Information Security Triad to Enhance the Proposed System Security Features}

The base station can detect the location of soldiers through Global positioning system (GPS), [8].

In order to enhance the proposed system security, Information security scheme provides many security measures that useful to guide policies for information security, to prevent unauthorized access to use, modify, record, and intercept the data. [6] CIA Triad schematic shown in figure 5.

Consists of three main parts Confidentiality, Integrity, Availability (CIA Triad). Starting with confidentiality includes sub elements Authentication, Authorization, and Encryption in this proposed system the Authorization: the base station website has a login form with password encrypted. Authorization: only authorized use can access with username and password. Encryption: the password is encrypted.

Integrity: ensure that the data no modified by any malicious users using hashing method to make sure that the data is the same by using hashing algorithms. Availability: the information available only to authorize user when needed using Firewall to prevent unwanted packets to go to the server and prevent the DOS attacks, not that but also using Access Control List (ACL) standard and extended to prevent unwanted protocol also an IP. [5]

SSL, TLS protocols used as another method to enhance the security in the proposed system. (TLS/SSL) security protocols used to secure data transferring between to machines. Integrity is addressed through the use of message authentication in each message from the first handshake. Additionally, non-repudiation is accounted for through certificate passing in addition to the integrity check from the message authentication. Though more responsibility for the Availability portion of the model (in this example) is placed on the server, Availability is slightly addressed since secure communications prevent malicious users from having direct access to the system. [6]

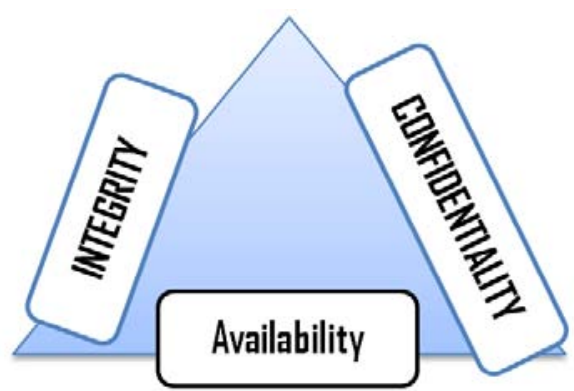

Figure 5. CIA Triad.

\section{Simulation Results}

Figures (from figure 6 to figure 8) represent the output of the GUI website interface of new simulation with three selected parts. Parameters that monitored (heartbeat, oxygen percentage, temperature). So base station can make statistics depending on these statistics base station can give the soldier the suitable decision. 


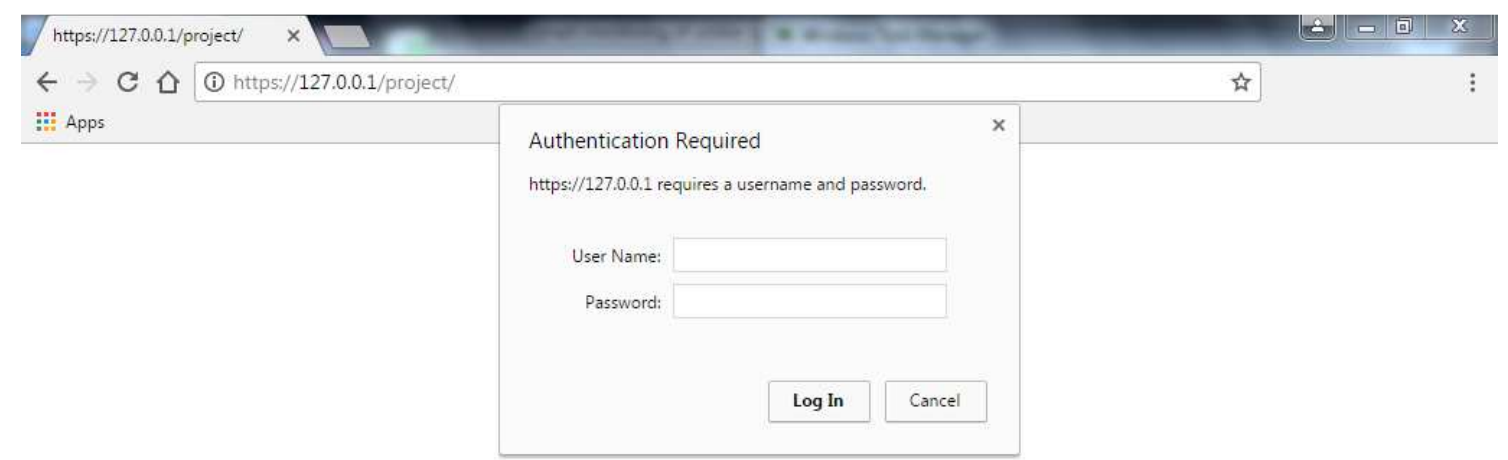

Figure 6. Login form appears to authorized user.

\section{$\because$ OPERATION 1}

\section{Save Hostages}

\section{CAPTAIN: Mostafa}

Lorem ipsum dolor sit amet, consectetur adipiscing elit. Etiam feugiat sodales ullamcorper. Sed et sollicitudin velit, eu hendrerit eros.

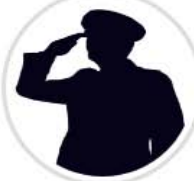

는 STATUS

\section{Monitoring Soldier}

\section{SOLDIER: Peter}

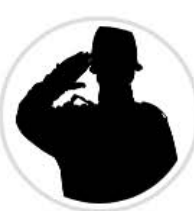

Lorem ipsum dolor sit amet, consectetur adipiscing elit. Etiam feugiat sodales ullamcorper. Sed et sollicitudin velit, eu hendrerit eros

Q STATUS

\section{Monitoring Soldier}

\section{Monitoring The Operation}

Figure 7. Base Station GUI displaying the current operation

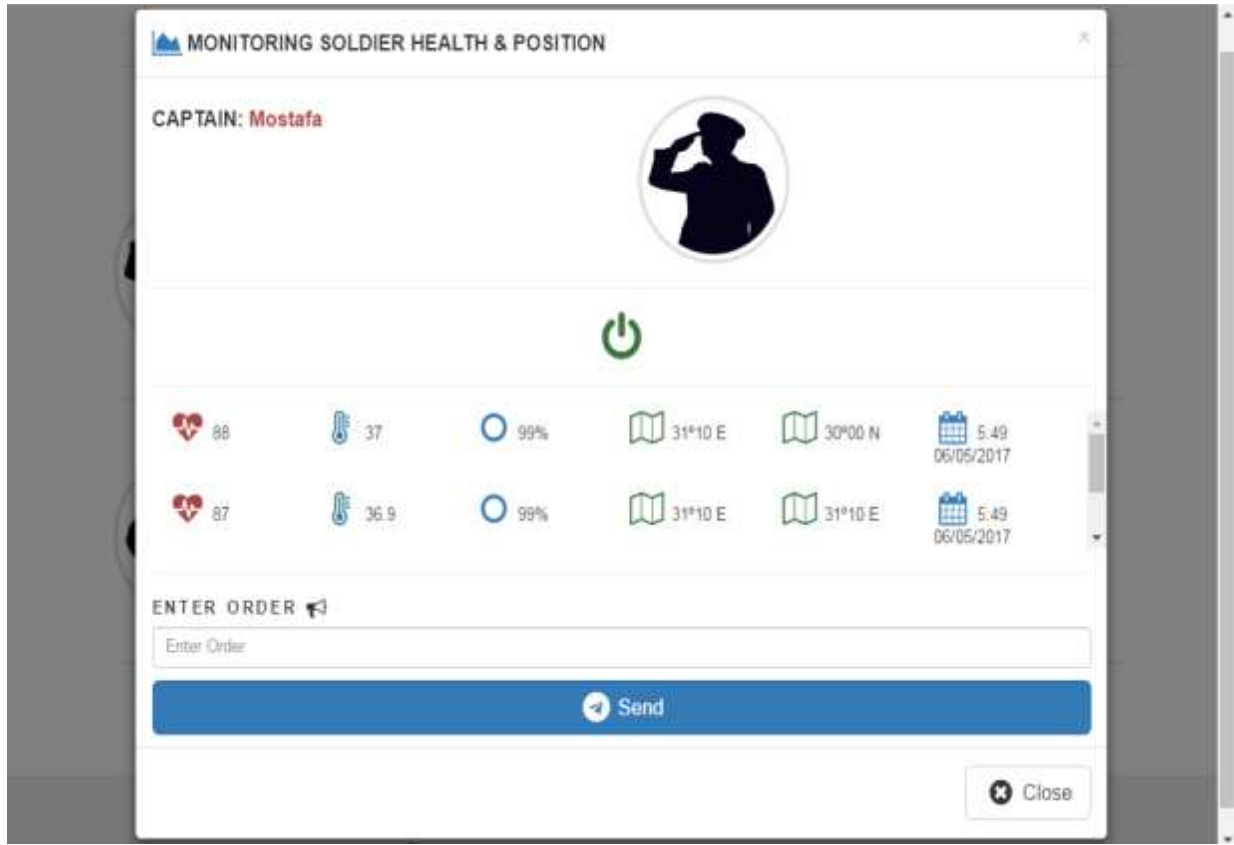

Figure 8. Base Station GUI displaying Soldier status. 


\section{Conclusion and Future Work}

This paper shade the light on this proposed system that will add an additional layer to protect soldiers from risks that they faces in the warfare from being injured or lost during the warfare. This proposed system provides real time smart monitoring system that easy to carry light weight, allow the Base Station to identify the soldier also locate its position and it's health condition continually this allows the Base Station to collect and analysis those information to plan and tactics the strategies, also to provide an accurate, fast and efficient medical treatment. Soldier can ask for help or supplies through SOS and get commands displayed in the LCD with vibration to notice it. This system special designed to suit the warfare situations not that but also for special operation.

\section{References}

[1] H. B. Lim, D. Ma, B. Wang, Z. Kalbarczyk, R. K. Iyer and K. L. Watkin, "A Soldier Health Monitoring System for Military Applications," 2010 International Conference on Body Sensor Networks, Singapore, 2010, pp. 246-249.

[2] Imperial Journal of Interdisciplinary Research (IJIR) Vol-2, Issue-4, 2016 ISSN: 2454-1362,.

[3] MD5 Algorithm (2016, Nov. 18). Library of Congress Government Site Available:http://id.loc.gov/vocabulary/preservation/cryptograp hicHashFunctions/md5.html [online].
[4] w3schools. Organization (2016, Nov. 10). The world's largest web developer site Available: http://www.w3schools.com/ [online].

[5] Journal of Information Security, 2016, 7, 185-194 Published Online April 2016 in SciRes. http://www.scirp.org/journal/jis http://dx.doi.org/10.4236/jis.2016.73014

[6] Journal of Advances in Computer Networks, Vol. 2, No. 3, September 2014.

[7] Hock Beng Lim, Di Ma, Bang Wang, Zbigniew Kalbarczyk, Ravishankar K. Iyer, Kenneth L. Watkin, "A Soldier Health Monitoring System for Military Applications", 2010 International Conference on Body Sensor Networks, 978-0-7695-4065-8/10/\$26.00 (C) 2010 IEEE, DOI: 10.1109/BSN.2010.58, pp: (246-249).

[8] William Walker, A. L. Praveen Aroul, Dinesh Bhatia, "Mobile Health Monitoring Systems", 31st Annual International Conference of the IEEE EMBS, Minneapolis, Minnesota, USA, September 2-6, 2009, 978-1-4244-3296-7/09/\$25.00 C 2009 IEEE, pp: (5199-5202).

[9] M. Pranav Sailesh, C. Vimal Kumar, B. Cecil, B. M. Mangal Deep, P. Sivraj, "Smart Soldier Assistance using WSN", International Conference on Embedded Systems - (ICES 2014), 978-1-4799-5026-3/14/\$31.00 (C) 2014 IEEE, pp: (244-249).

[10] P. S. Kurhe, S. S. Agrawal, "Real Time Tracking and Health Monitoring System of Remote Soldier Using ARM 7", International Journal of Engineering Trends and Technology, ISSN: 2231-5381, Volume 4, Issue 3, No. 1, March 2013, pp: (311-315). 